TRANSACTIONS OF THE

AMERICAN MATHEMATICAL SOCIETY

Volume 350, Number 7, July 1998, Pages 2847-2870

S $0002-9947(98) 02204-1$

\title{
KRUŽKOV'S ESTIMATES FOR SCALAR CONSERVATION LAWS REVISITED
}

\author{
F. BOUCHUT AND B. PERTHAME
}

\begin{abstract}
We give a synthetic statement of Kružkov-type estimates for multidimensional scalar conservation laws. We apply it to obtain various estimates for different approximation problems. In particular we recover for a model equation the rate of convergence in $h^{1 / 4}$ known for finite volume methods on unstructured grids.

Les estimations de Kružkov pour les lois de conservation scalaires revisitées

Résumé Nous donnons un énoncé synthétique des estimations de type de Kružkov pour les lois de conservation scalaires multidimensionnelles. Nous l'appliquons pour obtenir d'estimations nombreuses pour problèmes différents d'approximation. En particulier, nous retrouvons pour une équation modèle la vitesse de convergence en $h^{1 / 4}$ connue pour les méthodes de volumes finis sur des maillages non structurés.
\end{abstract}

\section{INTRODUCTION AND MAIN RESULTS}

This paper deals with error estimates for a multidimensional scalar conservation law

$$
\left\{\begin{array}{l}
\left.\partial_{t} v+\operatorname{div} f(v)=0 \quad \text { in } \quad\right] 0, \infty\left[\times \mathbb{R}^{N},\right. \\
v(0, x)=v^{0}(x) .
\end{array}\right.
$$

Here the flux function $f: \mathbb{R} \rightarrow \mathbb{R}^{N}$ is assumed to be Lipschitz continuous for simplicity. The equation has to be supplemented with the entropy inequalities, for any $S: \mathbb{R} \rightarrow \mathbb{R}$ convex and Lipschitz:

$$
\left.\partial_{t} S(v)+\operatorname{div} \eta(v) \leq 0 \quad \text { in } \quad\right] 0, \infty\left[\times \mathbb{R}^{N},\right.
$$

with $\eta^{\prime}=S^{\prime} f^{\prime}$. Throughout the paper, all partial differential equations and inequations are understood in the usual sense of distributions. After the works of P.D. Lax [12], O.A. Oleinik [15], A.I. Vol'pert [20], S.N. Kružkov [10], it is well-known that for any $v^{0} \in L_{l o c}^{1}\left(\mathbb{R}^{N}\right)$, there exists a unique solution $v \in C\left(\left[0, \infty\left[, L_{l o c}^{1}\left(\mathbb{R}^{N}\right)\right)\right.\right.$ to (1.1)-(1.2).

We are given an approximate solution $u$ and we wish to estimate the difference $u-v$. The famous method of S.N. Kružkov [10] has been used in many situations to obtain such estimates. It relies on a doubling of the variables and on a penalization

Received by the editors July 8, 1996.

1991 Mathematics Subject Classification. Primary 65M15, 35L65, 35B30.

Key words and phrases. Multi-dimensional scalar conservation laws, error estimates, entropy inequalities, finite volumes.

Mots-clés. Lois de conservation scalaires multidimensionnelles, estimations d'erreur, inégalités d'entropie, volumes finis. 
procedure. When applied to numerical approximations (such as finite volumes or finite elements), following the ideas of N.N. Kuznetsov [11], it becomes extremely intricate because the technicalities due to the large number of variables are added to notational difficulties for the numerical approximation itself. This is especially true for unstructured grids.

In this context, the derivation of the rate of convergence for first-order finite volumes methods, in several dimensions and for unstructured grids, is still complicated. On the other hand the final result, a convergence rate in $h^{1 / 4}$ rather than $h^{1 / 2}$ in one space dimension (see R. Sanders [17]), is very easy to explain. This loss just comes from the $B V$ estimate, which blows like $h^{-1 / 2}$ (while it is bounded in one dimension); see S. Champier, T. Gallouët, R. Herbin [3], B. Cockburn, F. Coquel, P. Le Floch [4][5], A. Szepessy [18], J.-P. Vila [19], B. Cockburn, P.-A. Gremaud [7], R. Eymard, T. Gallouët, R. Herbin [9]. For fluctuation splitting schemes this also applies; see B. Perthame [16].

In order to simplify these proofs, we propose to formalize Kružkov's method as follows. We assume that $u$ solves the entropy inequalities with error terms which are partial derivatives. For $k \in \mathbb{R}$

$$
\begin{aligned}
\partial_{t} \mid u & -k \mid+\operatorname{div} \operatorname{sgn}(u-k)[f(u)-f(k)] \\
& \left.\leq \partial_{t} G_{k}+\operatorname{div} H_{k}+K_{k}+\sum_{1 \leq i, j \leq N} \frac{\partial^{2} L_{k}^{(i j)}}{\partial x_{i} \partial x_{j}} \quad \text { in } \quad\right] 0, \infty\left[\times \mathbb{R}^{N},\right.
\end{aligned}
$$

where $G_{k}, H_{k}^{(j)}, K_{k}, L_{k}^{(i j)}$ are local Radon measures, and satisfy, in the sense of measures,

$$
\begin{array}{ll}
\left|G_{k}(t, x)\right| \leq \alpha_{G}(t, x), & \left|H_{k}^{(j)}(t, x)\right| \leq \alpha_{H}^{(j)}(t, x), \\
\left|K_{k}(t, x)\right| \leq \alpha_{K}(t, x), & \left|L_{k}^{(i j)}(t, x)\right| \leq \alpha_{L}^{(i j)}(t, x)
\end{array}
$$

with $\alpha_{G}, \alpha_{H}^{(j)}, \alpha_{K}, \alpha_{L}^{(i j)}$ are non-negative $k$-independent Radon measures.

Using Kružkov's method, we claim that one can estimate $\|u-v\|_{L^{1}}$ in terms of $\left\|\alpha_{G}\right\|_{\mathcal{M}_{t, x}},\left\|\alpha_{H}\right\|_{\mathcal{M}_{t, x}}$, etc. And it is the very striking and fundamental idea behind all the error estimates that the right estimates are always of the type

$$
\|u-v\|_{L^{1}} \leq C \sqrt{\left\|\sup _{k}\left|G_{k}\right|\right\|_{\mathcal{M}_{t, x}}}+C \sqrt{\left\|\sup _{k}\left|H_{k}\right|\right\|_{\mathcal{M}_{t, x}}}+\ldots
$$

(see the precise statement in Theorem 2.1). The derivatives in the error terms are paid, for the final estimate, only by a square root of the appropriate norm, which is taken in $\mathcal{M}_{t, x}\left(L_{k}^{\infty}\right)$. This is the main contribution of this paper, to indicate how to reduce the estimates to the form above.

More precisely, we claim that in many situations, it is not necessary to perform the doubling of variables explicitly - it is enough to use the abstract estimate of Theorem 2.1, and this greatly improves the understanding. This strategy was initiated in a paper by the authors and C. Bourdarias [2]. This approach is shown to be successful in the most classical situations, which we develop independently in $\S \S 3,4,5$. Moreover, we are able to improve the corresponding results. For multidimensional monotone finite volume methods, it is also possible to write an equation of the form (1.3) - see the recent paper of R. Eymard, T. Gallouët, R. Herbin [9]. 

of

In $\S 3$, we study the following problem. We assume that $u$ is an entropy solution

$$
\left.\partial_{t} u+\operatorname{div} g(u)=0 \quad \text { in } \quad\right] 0, \infty\left[\times \mathbb{R}^{N},\right.
$$

with $g$ another Lipschitz flux function. Then using Theorem 2.1, we are able to estimate $\|u-v\|_{L^{1}}$ in terms of $g-f$. We indeed recover the estimate in $\operatorname{lip}(g-f)$ that was used by C.M. Dafermos [8] and by B.J. Lucier [14] in the context of front tracking methods. But our formalism also enables to get a new estimate, in $\|g-f\|_{L^{\infty}}^{1 / 2}$. We refer to $\S 3$ for a precise statement.

In $\S 4$, we consider the nonlinear diffusion equation

$$
\left.\partial_{t} u+\operatorname{div} f(u)-\Delta \phi(u)=0 \quad \text { in } \quad\right] 0, \infty\left[\times \mathbb{R}^{N},\right.
$$

with $\phi: \mathbb{R} \rightarrow \mathbb{R}$ a Lipschitz and nondecreasing function. We refer to Ph. Benilan, R. Gariepy [1] for recent results on that equation. We here consider an entropy solution in the sense that for any $S: \mathbb{R} \rightarrow \mathbb{R}$ convex and Lipschitz,

$$
\left.\partial_{t} S(u)+\operatorname{div} \eta(u)-\Delta \nu(u) \leq 0 \quad \text { in } \quad\right] 0, \infty\left[\times \mathbb{R}^{N},\right.
$$

with $\eta^{\prime}=S^{\prime} f^{\prime}$ and $\nu^{\prime}=S^{\prime} \phi^{\prime}$. It is easy to see that it is equivalent to requiring that (1.7) holds for the entropies $S_{k}(\xi)=|\xi-k|, k \in \mathbb{R}$ :

$$
\left.\partial_{t}|u-k|+\operatorname{div} \operatorname{sgn}(u-k)[f(u)-f(k)]-\Delta|\phi(u)-\phi(k)| \leq 0 \quad \text { in } \quad\right] 0, \infty\left[\times \mathbb{R}^{N} .\right.
$$

Notice also that the equation (1.6) is recovered by letting $k \rightarrow \pm \infty$.

Our approach allows us to recover the usual estimate of $\|u-v\|_{L^{1}}$ in " $\varepsilon^{1 / 2}$ ", i.e., $\operatorname{lip}(\phi)^{1 / 2}$ for $v, u \in L_{t}^{\infty}\left(B V\left(\mathbb{R}^{N}\right)\right)$, and also the recent result of B. Cockburn, P.-A. Gremaud [6] which states that $v \in L_{t}^{\infty}\left(B V\left(\mathbb{R}^{N}\right)\right)$ and only $u \in L_{t}^{\infty}\left(L^{1}\left(\mathbb{R}^{N}\right)\right)$ is enough to get the same estimate. Moreover our result (see (ii) below) explains how the regularity of $\phi$ is involved in this matter, and we also give a new result for "irregular" diffusions $\phi$. We have the following results.

Theorem 1.1. Assume that $u \in L_{l o c}^{\infty}\left(\left[0, \infty\left[, L^{1}\left(\mathbb{R}^{N}\right)\right)\right.\right.$ is right-continuous with values in $L_{l o c}^{1}\left(\mathbb{R}^{N}\right)$ and is an entropy solution in the sense of (1.8). Let $v$ be an entropy solution of (1.1) with initial data $v^{0} \in L^{1} \cap B V\left(\mathbb{R}^{N}\right)$. Then, for any $T \geq 0$, we have:

(i) If $T V(u(t,)) \leq$.$V for any t \geq 0$, then

$$
\|u(T, .)-v(T, .)\|_{L^{1}} \leq\left\|u^{0}-v^{0}\right\|_{L^{1}}+C \sqrt{T V\left(v^{0}\right) V} \sqrt{T \operatorname{lip}(\phi)} .
$$

(ii) If $\|u(t, .)\|_{L^{1}} \leq U$ for any $t \geq 0$, then, denoting $Q=\sup _{\xi \neq 0}|\phi(\xi)-\phi(0)| /|\xi|$,

$$
\|u(T, .)-v(T, .)\|_{L^{1}} \leq\left\|u^{0}-v^{0}\right\|_{L^{1}}+C_{N} T V\left(v^{0}\right)^{2 / 3}(T Q U)^{1 / 3} .
$$

(iii) The following inequality holds:

$$
\|u(T, .)-v(T, .)\|_{L^{1}} \leq\left\|u^{0}-v^{0}\right\|_{L^{1}}+C T V\left(v^{0}\right) \sqrt{T \operatorname{lip}(\phi)} .
$$

Here and throughout this paper, the total variation $T V\left(v^{0}\right)$ of a function $v^{0} \in$ $B V\left(\mathbb{R}^{N}\right)$ is defined by

$$
T V\left(v^{0}\right)=\sum_{j=1}^{N} \int_{\mathbb{R}^{N}}\left|\frac{\partial v^{0}}{\partial x_{j}}\right| .
$$

The letter $C$ denotes various absolute constants, while $C_{N}$ denotes a possible dependence in the dimension $N$. 
Notice that if $v$ is bounded, say $a \leq v \leq b$, then the Lipschitz constant of $\phi$ in (1.11) can be replaced by lip $(\phi)$ by using Appendix A2 (ii) instead of (i) in the proof (see $\S 4)$.

$$
[a, b]
$$

For the sake of completeness, let us also recall that another classical analysis leading to local estimates in $\operatorname{lip}(\phi)^{1 / 4}$ can be carried out when $\phi(\xi)=\sigma \xi, \sigma>0$, by using the entropy dissipation equation (for $u^{0} \in L^{2}\left(\mathbb{R}^{N}\right)$ )

$$
\partial_{t} \frac{u^{2}}{2}+\operatorname{div} \eta(u)-\Delta\left(\sigma \frac{u^{2}}{2}\right)=-\sigma|\nabla u|^{2},
$$

with $\eta^{\prime}(\xi)=\xi f^{\prime}(\xi)$ (see [3], [18], [4], [5], [19], [7], [9] and $\S 5$ ). To deal with a general $\phi$, the inequalities (1.7) are not sufficient since the entropy dissipation is neglected.

In $\S 5$, we present a relaxation model towards piecewise constant functions. It does not involve notations as complicated as those of finite volumes, but leads to basically the same kind of theoretical difficulties. We show that error estimates can again be reduced to studying (1.3)-(1.4). We are given a general grid $\left(C_{i}\right)_{i \in I}$ of $\mathbb{R}^{N}: I$ is a countable set; $C_{i}$ is a Borel set for any $i \in I ;\left|C_{i} \cap C_{j}\right|=0$ for $i \neq j$; $\mathbb{R}^{N}=\mathcal{N} \cup \bigcup_{i \in I} C_{i}$ with $|\mathcal{N}|=0$; and

$$
h=\sup _{i \in I} \operatorname{diam}\left(C_{i}\right)<\infty .
$$

For technical reasons we assume that the following $L^{1}-B V$ condition is satisfied: there exists a constant $K_{h} \geq 0$ such that

$$
\forall i \in I \quad \mathbb{I}_{C_{i}} \in B V\left(\mathbb{R}^{N}\right), T V\left(\mathbb{I}_{C_{i}}\right) \leq K_{h}\left|C_{i}\right| .
$$

Notice that this is not a "regularity" assumption on the grid since $K_{h}$ is allowed to blow up very fast when $h \rightarrow 0$. It only means that there is no degeneracy at infinity. We define the piecewise constant projector $P^{0}: L_{l o c}^{1}\left(\mathbb{R}^{N}\right) \rightarrow L_{l o c}^{1}\left(\mathbb{R}^{N}\right)$ by

$$
\left(P^{0} u\right)_{\mid C_{i}}=\frac{1}{\left|C_{i}\right|} \int_{C_{i}} u
$$

We consider for a given $\varepsilon>0$ the unique solution $u \in C\left(\left[0, \infty\left[, L^{1}\left(\mathbb{R}^{N}\right)\right)\right.\right.$ of

$$
\left\{\begin{array}{c}
\left.\partial_{t} u+\operatorname{div} f(u)=\frac{P^{0} u-u}{\varepsilon} \text { in }\right] 0, \infty\left[\times \mathbb{R}^{N},\right. \\
u(0, .)=u^{0} \in L^{1}\left(\mathbb{R}^{N}\right),
\end{array}\right.
$$

with the entropy inequalities

$$
\left.\partial_{t} S(u)+\operatorname{div} \eta(u) \leq S^{\prime}(u) \frac{P^{0} u-u}{\varepsilon} \quad \text { in } \quad\right] 0, \infty\left[\times \mathbb{R}^{N}\right.
$$

for any $S$ convex, Lipschitz and $C^{1}$ (or equivalently for any entropy $S_{k}(\xi)=|\xi-k|$ ). Again we may estimate the difference from the exact solution.

Theorem 1.2. With the above notations and assumptions, if $u^{0} \in L^{1} \cap L^{2}\left(\mathbb{R}^{N}\right)$ then

$$
\iint_{0, T[\times \omega}\left|\frac{P^{0} u-u}{\varepsilon}\right| \leq \sqrt{\frac{T|\omega|}{2 \varepsilon}}\left\|u^{0}\right\|_{L^{2}}
$$

for any $T>0$ and any bounded subset $\omega$ of $\mathbb{R}^{N}$. Denoting by $v$ an entropy solution of (1.1) with initial data $v^{0} \in B V\left(\mathbb{R}^{N}\right)$, we have for any $T>0, x_{0} \in \mathbb{R}^{N}$ and 
$R>0$

$$
\begin{aligned}
& \int_{\left|x-x_{0}\right|<R}|u(T, x)-v(T, x)| d x \leq \int_{\left|x-x_{0}\right|<R+M T+\sqrt{\widehat{R} \ell}}\left|u^{0}(x)-v^{0}(x)\right| d x \\
& +C_{N} \sqrt{\ell}\left[T V\left(v^{0}\right) \sqrt{\widehat{R}}+\left\|u^{0}\right\|_{L^{2}} \widehat{R}^{N / 4-1 / 2}\left(\widehat{R}^{N / 4}+\ell^{N / 4}\right)\right]
\end{aligned}
$$

with $M=\operatorname{lip}(f), \widehat{R}=R+M T+h$ and $\ell=h \sqrt{T / \varepsilon}$.

In other words, the estimate is of order $h^{1 / 2} / \varepsilon^{1 / 4}$. When $\varepsilon \sim h$, which is similar to the limitation imposed by a CFL condition for finite volumes, we recover the usual rate in $h^{1 / 4}$. However, the estimate (1.18) is not really interesting because we have in mind to approximate the solution $v$ by a piecewise constant function. Thus, it is more appropriate to estimate $P^{0} u-v$. This can be performed by combining (1.17) and (1.18), and yields an estimate in $L^{1}(] 0, T\left[\times B\left(x_{0}, R\right)\right)$.

Notice also that it is very natural to choose $u^{0}=P^{0} v^{0}$. Then, assuming that $v^{0} \in L^{1} \bigcap L^{2} \bigcap B V\left(\mathbb{R}^{N}\right)$, the $L^{2}$ norm of $u^{0}$ in (1.18) can be majorized by $\left\|v^{0}\right\|_{L^{2}}$. Thus, if the grid is regular in the sense that $C_{i}$ is open and convex, and

$$
\operatorname{diam}\left(C_{i}\right)^{N+1} \leq \kappa\left|C_{i}\right| h,
$$

then the initial error can be estimated by

$$
\left\|u^{0}-v^{0}\right\|_{L^{1}\left(\mathbb{R}^{N}\right)} \leq C_{N} \kappa T V\left(v^{0}\right) h .
$$

This is just a straightforward application of the Poincaré-Wirtinger inequality for a convex domain.

The paper is organized as follows. In $\S 2$ we state and prove our abstract version of Kružkov's estimates. In $\S \S 3,4$ and 5 we treat in detail the above independent applications. The appendix is devoted to two independent lemmas. The first deals with a special "inverse" of the "div" operator which furnishes the link between finite volumes and the formulation (1.3). It is needed for the proof of Theorem 1.2. The second deals with a general estimate on the gradient $\nabla[g(u)]$, where $g$ is Lipschitz and $u \in B V$. It is used frequently in this paper.

\section{An ERror EStimate For PARTIAL DERIVATIVES IN THE RIGHT-HAND Side}

This section is devoted to the main estimate of this paper, the reformulation of Kružkov type estimates. We give two versions of the result.

Theorem 2.1. Let $u, v \in L_{\text {loc }}^{\infty}\left(\left[0, \infty\left[, L_{l o c}^{1}\left(\mathbb{R}^{N}\right)\right)\right.\right.$ be right-continuous with values in $L_{\text {loc }}^{1}\left(\mathbb{R}^{N}\right)$. Assume that $u$ solves the entropy inequalities with right-hand side (1.3)-(1.4), and that $v$ is an exact solution: for $k \in \mathbb{R}$

$$
\left.\partial_{t}|v-k|+\operatorname{div} \operatorname{sgn}(v-k)[f(v)-f(k)] \leq 0 \quad \text { in } \quad\right] 0, \infty\left[\times \mathbb{R}^{N} .\right.
$$

Moreover, assume that

$$
\alpha_{G} \in L_{l o c}^{\infty}\left(\left[0, \infty\left[, L_{l o c}^{1}\left(\mathbb{R}^{N}\right)\right) .\right.\right.
$$

Then, for any $T \geq 0, x_{0} \in \mathbb{R}^{N}, R>0, \Delta>0, \delta>0, \nu \geq 0$, denoting 


$$
M=\operatorname{lip}(f), \quad B_{t}=B\left(x_{0}, R+M(T-t)+\Delta+\nu\right),
$$

we have

$$
\begin{aligned}
\int_{\left|x-x_{0}\right|<R} & |u(T, x)-v(T, x)| d x \\
& \leq \int_{B_{0}}|u(0, x)-v(0, x)| d x+C\left(E^{t}+E^{x}+E^{G}+E^{H}+E^{K}+E^{L}\right),
\end{aligned}
$$

with $C$ an absolute constant and

$$
\begin{gathered}
E^{t}=\sup _{\substack{0<s-t<\delta \\
t=0 \text { or } T}} \int_{B_{t}}|v(s, x)-v(t, x)| d x, \\
E^{x}=\sup _{\substack{|h|<\Delta \\
t=0 \text { or } T}} \int_{B_{t}}|v(t, x+h)-v(t, x)| d x, \\
E^{K}=\iint_{\substack{x \in B_{t} \\
0<t \leq T}} \alpha_{K}(t, x), \quad E^{H}=\frac{1}{\Delta} \sum_{j=1}^{N} \iint_{\substack{x \in B_{t} \\
0<t \leq T}} \alpha_{H}^{(j)}(t, x), \\
E^{L}=\frac{1}{\Delta^{2}} \sum_{1 \leq i, j \leq N} \iint_{\substack{x \in B_{t} \\
0<t \leq T}} \alpha_{L}^{(i j)}(t, x), \\
E^{G}=\left(1+\frac{T}{\delta}+\frac{M T}{\Delta+\nu}\right) \sup _{0<t<2 T} \int_{B_{t}} \alpha_{G}(t, x) d x .
\end{gathered}
$$

Before we prove Theorem 2.1, some comments are in order.

1. If $u$ is an exact solution, the right-hand side of (1.3) is zero. Hence, only the error terms $E^{t}$ and $E^{x}$ remain in (2.4). Therefore, we can choose $\nu=0$ and let $\delta, \Delta \rightarrow 0$ so that by the assumed regularity of $v$ we get $E^{t}, E^{x} \rightarrow 0$. We hence recover Kružkov's estimate [10]

$$
\int_{\left|x-x_{0}\right|<R}|u(T, x)-v(T, x)| d x \leq \int_{\left|x-x_{0}\right|<R+M T}|u(0, x)-v(0, x)| d x .
$$

When the right-hand side of (1.3) is not zero, we can use the well-known TVD property of the exact solution $v$. If $v^{0} \in B V\left(\mathbb{R}^{N}\right)$, we obtain

$$
E^{t} \leq M T V\left(v^{0}\right) \delta, \quad E^{x} \leq T V\left(v^{0}\right) \Delta .
$$

Then, we choose the time and space regularization parameters $\delta$ and $\Delta$ so as to minimize the error terms in (2.4). The parameter $\nu$ either is chosen to be 0 for a local estimate or tends to $+\infty$ for a global one.

2. Our assumption that $u$ and $v$ are $L_{l o c}^{1}$ right-continuous is motivated by numerical schemes; it is well suited for such problems. We refer to T.-P. Liu, M. Pierre 
[13] for uniqueness results in one dimension when the initial data is recovered only in weak $L^{1}$.

3. For numerical schemes, the assumption (2.2) on $\alpha_{G}$ is somehow related to an estimate on the time modulus of continuity of $u$. In fact, $\alpha_{G}$ is comparable with $\Delta t \partial_{t} u$, with $\Delta t$ the time step (see [2]). However, when no such estimate is available, it is possible to get an estimate of $\|u-v\|_{L_{t, x}^{1}}$ with only the regularity of $\alpha_{G}$ stated in (1.4) and an estimate of $\alpha_{G}$ only for small $t$. In order to obtain this result, just average (2.4) with respect to $T$ and then observe that when averaged, the last inequality of (2.28) in the proof below can be replaced by a suitable one since $\left|\chi^{\prime}(t)\right| \leq Y_{\varepsilon}^{\prime}(t)+Y_{\varepsilon}^{\prime}(t-T)$. It is also possible to choose a different test function $\chi$, see R. Eymard, T. Gallouët, R. Herbin [9].

Proof of Theorem 2.1. Let us introduce two test functions, $\Phi \in C_{c}^{\infty}(] 0, \infty\left[\times \mathbb{R}^{N}\right)$, $\Phi \geq 0$, and $\zeta \in C_{c}^{\infty}(]-\infty, 0\left[\times \mathbb{R}^{N}\right), \zeta \geq 0$, to be chosen later on, and set

$$
\varphi(t, x, s, y)=\Phi(t, x) \zeta(t-s, x-y) .
$$

This choice is slightly different from that of S.N. Kružkov in [10], and is inspired by that of N.N. Kuznetsov [11] (who takes $\Phi=\mathbb{I}_{0<t<T}$ ). Notice that $\varphi \in C_{c}^{\infty}\left((] 0, \infty\left[\times \mathbb{R}^{N}\right)^{2}\right)$.

For each $(s, y) \in] 0, \infty\left[\times \mathbb{R}^{N}\right.$ and $k \in \mathbb{R}$, let us take $\varphi$ as a test function in $(t, x)$ for (1.3). We get with (1.4)

$$
\begin{aligned}
& -\iint_{] 0, \infty\left[\times \mathbb{R}^{N}\right.}\left[|u-k| \partial_{t} \varphi+\operatorname{sgn}(u-k)[f(u)-f(k)] \cdot \nabla_{x} \varphi\right](t, x) d t d x \\
& \leq \iint_{0, \infty\left[\times \mathbb{R}^{N}\right.}\left[-G_{k} \partial_{t} \varphi-H_{k} \cdot \nabla_{x} \varphi+K_{k} \varphi+\sum_{1 \leq i, j \leq N} L_{k}^{(i j)} \frac{\partial^{2} \varphi}{\partial x_{i} \partial x_{j}}\right](t, x) d t d x \\
& \leq \iint_{] 0, \infty\left[\times \mathbb{R}^{N}\right.}\left[\alpha_{G}\left|\partial_{t} \varphi\right|+\sum_{j=1}^{N} \alpha_{H}^{(j)}\left|\frac{\partial \varphi}{\partial x_{j}}\right|+\alpha_{K} \varphi+\sum_{1 \leq i, j \leq N} \alpha_{L}^{(i j)}\left|\frac{\partial^{2} \varphi}{\partial x_{i} \partial x_{j}}\right|\right](t, x) d t d x .
\end{aligned}
$$

Similarly, for each $(t, x) \in] 0, \infty\left[\times \mathbb{R}^{N}\right.$ and $l \in \mathbb{R}$, we take $\varphi$ as a test function in $(s, y)$ for $(2.1)$, and obtain

$$
-\iint_{] 0, \infty\left[\times \mathbb{R}^{N}\right.}\left[|v-l| \partial_{s} \varphi+\operatorname{sgn}(v-l)[f(v)-f(l)] \cdot \nabla_{y} \varphi\right](s, y) d s d y \leq 0 .
$$

Now we take $k=v(s, y)$ in (2.11) and integrate with respect to $(s, y)$; then we take $l=u(t, x)$ in $(2.12)$ and integrate with respect to $(t, x)$. By summing up the results we obtain 


$$
\begin{aligned}
& -\iiint \int\left[|u(t, x)-v(s, y)| \partial_{t} \Phi(t, x)\right. \\
& \left.\quad+\operatorname{sgn}(u(t, x)-v(s, y))[f(u(t, x))-f(v(s, y))] \cdot \nabla_{x} \Phi(t, x)\right] \\
& \quad \zeta(t-s, x-y) d s d t d x d y \\
& \leq \iiint \int\left[\alpha_{G}(t, x)\left|\partial_{t} \Phi(t, x) \zeta(t-s, x-y)+\Phi(t, x) \partial_{t} \zeta(t-s, x-y)\right|\right. \\
& \quad+\sum_{j=1}^{N} \alpha_{H}^{(j)}(t, x)\left|\partial_{j} \Phi(t, x) \zeta(t-s, x-y)+\Phi(t, x) \partial_{j} \zeta(t-s, x-y)\right| \\
& \quad+\alpha_{K}(t, x) \Phi(t, x) \zeta(t-s, x-y) \\
& \quad+\sum_{1 \leq i, j \leq N} \alpha_{L}^{(i j)}(t, x) \mid \partial_{i j}^{2} \Phi(t, x) \zeta(t-s, x-y)+\partial_{i} \Phi(t, x) \partial_{j} \zeta(t-s, x-y) \\
& \left.\quad+\partial_{j} \Phi(t, x) \partial_{i} \zeta(t-s, x-y)+\Phi(t, x) \partial_{i j}^{2} \zeta(t-s, x-y) \mid\right] d s d t d x d y \\
& \equiv R^{\alpha} .
\end{aligned}
$$

Let us now make the choice of $\Phi$ precise. Let $\theta>0$ and set $Y_{\theta}(t)=\int_{-\infty}^{t} Y_{\theta}^{\prime}(s) d s$ with $Y_{\theta}^{\prime}(t)=\frac{1}{\theta} Y_{1}^{\prime}\left(\frac{t}{\theta}\right)$ and $Y_{1}^{\prime} \in C_{c}^{\infty}(] 0,1[), Y_{1}^{\prime} \geq 0$ and $\int Y_{1}^{\prime}=1$.

We introduce another parameter $\varepsilon>0$ and a function $\chi \in C_{c}^{\infty}(] 0, T+\varepsilon[), \chi \geq 0$, to be chosen later. We define

$$
\begin{gathered}
\Phi(t, x)=\chi(t) \psi(t, x), \\
\psi(t, x)=1-Y_{\theta}\left(\left|x-x_{0}\right|-R-\Delta / 2-M(T-t)\right) \geq 0 .
\end{gathered}
$$

Then $\Phi \in C^{\infty}\left(\mathbb{R} \times \mathbb{R}^{N}\right)$ as soon as $M \varepsilon \leq R+\Delta / 2$. We have

$$
\begin{gathered}
\partial_{t} \Phi(t, x)=\chi^{\prime}(t) \psi(t, x)-M \chi(t) Y_{\theta}^{\prime}(), \\
\nabla_{x} \Phi(t, x)=-\chi(t) Y_{\theta}^{\prime}() \frac{x-x_{0}}{\left|x-x_{0}\right|} .
\end{gathered}
$$

Therefore, by the Lipschitz condition on $f$ we get

$$
\begin{gathered}
|u(t, x)-v(s, y)| \partial_{t} \Phi(t, x) \\
\quad+\operatorname{sgn}(u(t, x)-v(s, y))[f(u(t, x))-f(v(s, y))] \cdot \nabla_{x} \Phi(t, x) \\
=|u(t, x)-v(s, y)| \chi^{\prime}(t) \psi(t, x)-\chi(t) Y_{\theta}^{\prime}()[M|u(t, x)-v(s, y)| \\
\left.\quad+\operatorname{sgn}(u(t, x)-v(s, y))[f(u(t, x))-f(v(s, y))] \cdot \frac{x-x_{0}}{\left|x-x_{0}\right|}\right] \\
\leq|u(t, x)-v(s, y)| \chi^{\prime}(t) \psi(t, x) .
\end{gathered}
$$

Together with (2.13), this yields

$$
-\iiint \int|u(t, x)-v(s, y)| \chi^{\prime}(t) \psi(t, x) \zeta(t-s, x-y) d s d t d x d y \leq R^{\alpha} .
$$

Now, by the triangle inequality we get

$$
0 \leq I+R^{t}+R^{x}+R^{\alpha}
$$


with

$$
\begin{aligned}
I & =\iiint \int|u(t, x)-v(t, x)| \chi^{\prime}(t) \psi(t, x) \zeta(t-s, x-y) d s d t d x d y, \\
R^{t} & =\iiint \int|v(t, y)-v(s, y)|\left|\chi^{\prime}(t)\right| \psi(t, x) \zeta(t-s, x-y) d s d t d x d y, \\
R^{x} & =\iiint \int|v(t, x)-v(t, y)|\left|\chi^{\prime}(t)\right| \psi(t, x) \zeta(t-s, x-y) d s d t d x d y .
\end{aligned}
$$

We now prescribe $\zeta$ to be a regularizing sequence

$$
\begin{gathered}
\zeta(t, x)=\zeta^{t}(t) \zeta^{x}(x), \quad \zeta^{t}, \zeta^{x} \in C_{c}^{\infty}, \geq 0, \quad \int \zeta^{t} d t=\int \zeta^{x} d x=1, \\
\left.\zeta^{t}(t)=\frac{1}{\delta} \zeta_{1}^{t}\left(\frac{t}{\delta}\right), \quad \operatorname{supp} \zeta_{1}^{t} \subset\right]-1,0[ \\
\zeta^{x}(x)=\frac{1}{\Delta^{N}} \zeta_{1}^{x}\left(\frac{x}{\Delta}\right), \quad \operatorname{supp} \zeta_{1}^{x} \subset B(0,1 / 4) .
\end{gathered}
$$

We also choose $\zeta^{x}$ to be a product $\zeta_{1}^{x}(x)=\prod_{j=1}^{N} \zeta_{1}^{x_{j}}\left(x_{j}\right)$. For $\chi$ we take

$$
\chi(t)=Y_{\varepsilon}(t)-Y_{\varepsilon}(t-T)
$$

so that $0 \leq \chi \leq 1$. We are going to take the $\limsup _{\varepsilon \rightarrow 0}$ in (2.18), $\theta$ fixed.

We notice that

$$
\mathbb{I}_{\left|x-x_{0}\right|<R+M(T-t)+\Delta / 2} \leq \psi(t, x) \leq \mathbb{I}_{\left|x-x_{0}\right|<R+M(T-t)+\Delta / 2+\theta},
$$

and obtain

$$
R^{t} \leq \int_{\left|y-x_{0}\right|<R+M(T-t)+3 \Delta / 4+\theta}|v(t, y)-v(s, y)|\left(Y_{\varepsilon}^{\prime}(t)+Y_{\varepsilon}^{\prime}(t-T)\right) \zeta^{t}(t-s) d s d t d y .
$$

Then by right continuity of $v$ at 0 and $T$,

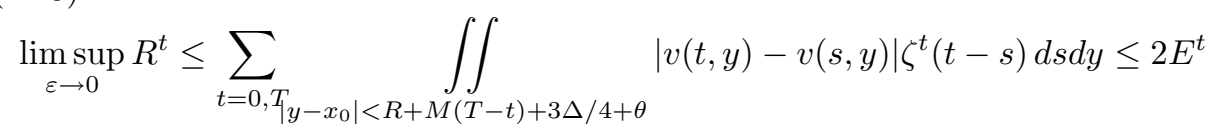

just by choosing $\theta=\Delta / 4+\nu$.

For the term $R^{x}$ we have

$$
R^{x} \leq \iiint_{\left|x-x_{0}\right|<R+M(T-t)+\Delta / 2+\theta}|v(t, x)-v(t, y)|\left(Y_{\varepsilon}^{\prime}(t)+Y_{\varepsilon}^{\prime}(t-T)\right) \zeta^{x}(x-y) d t d x d y,
$$

and by the same continuity property of $v$

$$
\begin{aligned}
\limsup _{\varepsilon \rightarrow 0} R^{x} & \leq \sum_{t=0, T} \iint_{\left|x-x_{0}\right|<R+M(T-t)+\Delta / 2+\theta}|v(t, x)-v(t, y)| \zeta^{x}(x-y) d x d y \\
& \leq 2 E^{x} .
\end{aligned}
$$


For $I$ we have, using (2.22),

$$
\begin{aligned}
I & =\iint|u(t, x)-v(t, x)|\left[Y_{\varepsilon}^{\prime}(t)-Y_{\varepsilon}^{\prime}(t-T)\right] \psi(t, x) d t d x \\
& \leq \int_{\left|x-x_{0}\right|<R+M(T-t)+\Delta / 2+\theta}|u(t, x)-v(t, x)| Y_{\varepsilon}^{\prime}(t) d t d x \\
& -\quad \iint_{\left|x-x_{0}\right|<R+M(T-t)+\Delta / 2}|u(t, x)-v(t, x)| Y_{\varepsilon}^{\prime}(t-T) d t d x,
\end{aligned}
$$

and by right continuity of $u$ and $v$ at 0 and $T$,

$$
\begin{aligned}
\limsup _{\varepsilon \rightarrow 0} I \leq & \int_{\left|x-x_{0}\right|<R+M T+\Delta / 2+\theta}|u(0, x)-v(0, x)| d x \\
& -\int_{\left|x-x_{0}\right|<R+\Delta / 2}|u(T, x)-v(T, x)| d x .
\end{aligned}
$$

It now remains to estimate $R^{\alpha}$ defined in (2.13). Using the bounds

$$
\left|\nabla_{x} \Phi(t, x)\right| \leq \frac{C}{\theta}, \quad\left|\partial_{t} \Phi(t, x)\right| \leq\left|\chi^{\prime}(t)\right|+C \frac{M}{\theta},
$$

and denoting by $\Omega$ the set

$$
\Omega=\left\{t, x ; \quad 0<t<T+\varepsilon, \quad\left|x-x_{0}\right|<R+M(T-t)+\Delta / 2+\theta\right\},
$$

we get the estimates

$$
\begin{gathered}
\iiint \int \alpha_{K}(t, x) \Phi(t, x) \zeta(t-s, x-y) \leq \iint_{\Omega} \alpha_{K}(t, x), \\
\iiint \int \alpha_{H}^{(j)}(t, x) \Phi(t, x)\left|\partial_{j} \zeta(t-s, x-y)\right| \leq \frac{C}{\Delta} \iint_{\Omega} \alpha_{H}^{(j)}(t, x), \\
\iiint \int \alpha_{G}(t, x) \Phi(t, x)\left|\partial_{t} \zeta(t-s, x-y)\right| \leq \frac{C}{\delta} \iint_{\Omega} \alpha_{G}(t, x), \\
\iiint \int \alpha_{H}^{(j)}(t, x)\left|\partial_{j} \Phi(t, x)\right| \zeta(t-s, x-y) \leq \frac{C}{\theta} \iint_{\Omega} \alpha_{H}^{(j)}(t, x), \\
\iiint \int \alpha_{G}(t, x)\left|\partial_{t} \Phi(t, x)\right| \zeta(t-s, x-y) \\
\leq C(1+M(T+\varepsilon) / \theta) \sup _{0<t<T+\varepsilon} \int_{\left|x-x_{0}\right|<R+M(T-t)+\Delta / 2+\theta} \alpha_{G}(t, x) d x .
\end{gathered}
$$

For the second-order terms of $R^{\alpha}$, we notice that in the support of $\nabla_{x} \Phi(t, x)$ we have $\left|x-x_{0}\right| \geq R+\Delta / 2-M \varepsilon+C_{0} \theta \geq C_{0} \theta$ for some $C_{0}>0$. This yields

$$
\left|\frac{\partial^{2} \Phi}{\partial x_{i} \partial x_{j}}\right| \leq \frac{C}{\theta^{2}}
$$


We hence get

(2.30)

$$
\begin{aligned}
\iiint \int \alpha_{L}^{(i j)}(t, x) \Phi(t, x)\left|\partial_{i j}^{2} \zeta(t-s, x-y)\right| & \leq \frac{C}{\Delta^{2}} \iint_{\Omega} \alpha_{L}^{(i j)}(t, x), \\
\iiint \int \alpha_{L}^{(i j)}(t, x)\left|\partial_{i} \Phi(t, x)\right|\left|\partial_{j} \zeta(t-s, x-y)\right| & \leq \frac{C}{\Delta \theta} \iint_{\Omega} \alpha_{L}^{(i j)}(t, x), \\
\iiint \int \alpha_{L}^{(i j)}(t, x)\left|\partial_{i j}^{2} \Phi(t, x)\right| \zeta(t-s, x-y) & \leq \frac{C}{\theta^{2}} \iint_{\Omega} \alpha_{L}^{(i j)}(t, x) .
\end{aligned}
$$

Taking into account that $\theta=\Delta / 4+\nu$, we obtain, by combining (2.28) and (2.30),

$$
\limsup _{\varepsilon \rightarrow 0} R^{\alpha} \leq C\left(E^{G}+E^{H}+E^{K}+E^{L}\right),
$$

and the $\lim \sup _{\varepsilon \rightarrow 0}$ in (2.18) together with the estimates (2.23), (2.14), (2.25) gives the result.

Notice that in Theorem 2.1 and its proof, we never use any specific property of the exact solution $v$. We only use the entropy inequalities (2.1) in the distributional sense for any $k \in \mathbb{R}$. Therefore, our result actually contains Kružkov's uniqueness theorem with the stated regularity. Moreover, it is possible to modify the proof in order to also consider error terms for $v$. The result is the following.

Proposition 2.2. We make the same assumptions as in Theorem 2.1, except that we replace the inequalities (2.1) on $v$ by

$$
\begin{aligned}
\partial_{t}|v-k| & +\operatorname{div} \operatorname{sgn}(v-k)[f(v)-f(k)] \\
& \left.\leq \partial_{t} \bar{G}_{k}+\operatorname{div} \bar{H}_{k}+\bar{K}_{k}+\sum_{1 \leq i, j \leq N} \frac{\partial^{2} \bar{L}_{k}^{(i j)}}{\partial x_{i} \partial x_{j}} \text { in }\right] 0, \infty\left[\times \mathbb{R}^{N},\right.
\end{aligned}
$$

where $\bar{G}_{k}, \bar{H}_{k}^{(j)}, \bar{K}_{k}, \bar{L}_{k}^{(i j)}$ are local measures, and

$$
\left|\bar{G}_{k}\right| \leq \beta_{G}, \quad\left|\bar{H}_{k}^{(j)}\right| \leq \beta_{H}^{(j)}, \quad\left|\bar{K}_{k}\right| \leq \beta_{K}, \quad\left|\bar{L}_{k}^{(i j)}\right| \leq \beta_{L}^{(i j)},
$$

with $\beta_{G}, \beta_{H}^{(j)}, \beta_{K}, \beta_{L}^{(i j)}$ being $k$-independent non-negative measures.

Then we have the same estimate (2.4), with the additional terms

$$
C\left(E^{\bar{G}}+E^{\bar{H}}+E^{\bar{K}}+E^{\bar{L}}\right),
$$

where

$$
\begin{gathered}
E^{\bar{K}}=\iint_{\substack{x \in B_{0} \\
0<t \leq T+\delta}} \beta_{K}(t, x), \quad E^{\bar{H}}=\frac{1}{\Delta} \sum_{j=1}^{N} \iint_{\substack{x \in B_{0} \\
0<t \leq T+\delta}} \beta_{H}^{(j)}(t, x), \\
E^{\bar{L}}=\frac{1}{\Delta^{2}} \sum_{1 \leq i, j \leq N} \iint_{\substack{x \in B_{0} \\
0<t \leq T+\delta}} \beta_{L}^{(i j)}(t, x), \\
E^{\bar{G}}=\frac{1}{\delta} \iint_{\substack{x \in B_{0} \\
0<t \leq T+\delta}} \beta_{G}(t, x) .
\end{gathered}
$$


Proof. The inequality (2.12) becomes

$$
\begin{aligned}
& -\iint_{] 0, \infty\left[\times \mathbb{R}^{N}\right.}\left[|v-l| \partial_{s} \varphi+\operatorname{sgn}(v-l)[f(v)-f(l)] \cdot \nabla_{y} \varphi\right](s, y) d s d y \\
& \leq \iint_{] 0, \infty\left[\times \mathbb{R}^{N}\right.}\left[-\bar{G}_{l} \partial_{s} \varphi-\bar{H}_{l} \cdot \nabla_{y} \varphi+\bar{K}_{l} \varphi+\sum_{1 \leq i, j \leq N} \bar{L}_{l}^{(i j)} \frac{\partial^{2} \varphi}{\partial y_{i} \partial y_{j}}\right](s, y) d s d y \\
& \leq \iint_{] 0, \infty\left[\times \mathbb{R}^{N}\right.}\left[\beta_{G}\left|\partial_{s} \varphi\right|+\sum_{j=1}^{N} \beta_{H}^{(j)}\left|\frac{\partial \varphi}{\partial y_{j}}\right|+\beta_{K} \varphi+\sum_{1 \leq i, j \leq N} \beta_{L}^{(i j)}\left|\frac{\partial^{2} \varphi}{\partial y_{i} \partial y_{j}}\right|\right](s, y) d s d y,
\end{aligned}
$$

and gives a new term in (2.13).

$$
\begin{aligned}
& R^{\beta}=\iiint \int\left[\beta_{G}(s, y) \Phi(t, x)\left|\partial_{t} \zeta(t-s, x-y)\right|\right. \\
&+\sum_{j=1}^{N} \beta_{H}^{(j)}(s, y) \Phi(t, x)\left|\partial_{j} \zeta(t-s, x-y)\right| \\
&+\beta_{K}(s, y) \Phi(t, x) \zeta(t-s, x-y) \\
&\left.+\sum_{1 \leq i, j \leq N} \beta_{L}^{(i j)}(s, y) \Phi(t, x)\left|\partial_{i j}^{2} \zeta(t-s, x-y)\right|\right] d s d t d x d y .
\end{aligned}
$$

Since where $\Phi(t, x) \zeta(t-s, x-y) \neq 0$ we have $(s, y) \in \widetilde{\Omega}$, with

$$
\widetilde{\Omega}=\left\{s, y ; \quad 0<s<T+\varepsilon+\delta, \quad\left|y-x_{0}\right|<R+M T+3 \Delta / 4+\theta\right\},
$$

we get

$$
\begin{aligned}
R^{\beta} \leq & \frac{C}{\delta} \iint_{\widetilde{\Omega}} \beta_{G}(s, y) \\
& +\frac{C}{\Delta} \sum_{j=1}^{N} \iint_{\widetilde{\Omega}} \beta_{H}^{(j)}(s, y)+\iint_{\widetilde{\Omega}} \beta_{K}(s, y)+\frac{C}{\Delta^{2}} \sum_{1 \leq i, j \leq N} \iint_{\widetilde{\Omega}} \beta_{L}^{(i j)}(s, y) .
\end{aligned}
$$

Therefore, $\limsup _{\varepsilon \rightarrow 0} R^{\beta} \leq C\left(E^{\bar{G}}+E^{\bar{H}}+E^{\bar{K}}+E^{\bar{L}}\right)$, and we get the result.

\section{Estimates For tWo DIFFERENT FLUX FUnCTIONS}

In this section we apply Theorem 2.1 to prove various estimates for the following problem. Given two globally Lipschitz flux functions $f, g: \mathbb{R} \rightarrow \mathbb{R}^{N}$ and $u^{0}, v^{0} \in$ $L_{l o c}^{1}\left(\mathbb{R}^{N}\right)$, we consider the entropy solutions $u$ and $v$ of

$$
\left.\partial_{t} u+\operatorname{div} g(u)=0, \quad \partial_{t} v+\operatorname{div} f(v)=0 \quad \text { in } \quad\right] 0, \infty\left[\times \mathbb{R}^{N}\right.
$$

with initial data $u^{0}$ and $v^{0}$ respectively. Then, what kind of estimate can we expect for $u-v$ in terms of $g-f$ ? We have the following result. 
Theorem 3.1. Let $v^{0} \in B V\left(\mathbb{R}^{N}\right)$. Then, with the above notations, for any $T \geq 0$ :

(i) The following estimate holds:

$$
\|u(T, .)-v(T, .)\|_{L^{1}\left(\mathbb{R}^{N}\right)} \leq\left\|u^{0}-v^{0}\right\|_{L^{1}\left(\mathbb{R}^{N}\right)}+C T V\left(v^{0}\right) T \operatorname{lip}(g-f) .
$$

(ii) Moreover,

$$
\begin{aligned}
& \|u(T, .)-v(T, .)\|_{L^{1}\left(\mathbb{R}^{N}\right)} \\
& \quad \leq\left\|u^{0}-v^{0}\right\|_{L^{1}\left(\mathbb{R}^{N}\right)}+C_{N}\left(\left\|u^{0}\right\|_{L^{1}\left(\mathbb{R}^{N}\right)} T V\left(v^{0}\right) T Q(f, g)\right)^{1 / 2},
\end{aligned}
$$

with

$$
Q(f, g)=\sup _{\xi \in \mathbb{R} \backslash\{0\}}|g(\xi)-f(\xi)-g(0)+f(0)| /|\xi| .
$$

(iii) If $g-f \in L^{\infty}(\mathbb{R})$, then for $x_{0} \in \mathbb{R}^{N}$ and $R>0$

$$
\begin{aligned}
& \int_{\left|x-x_{0}\right|<R}|u(T, x)-v(T, x)| \leq \int_{\left|x-x_{0}\right|<R+M T}\left|u^{0}(x)-v^{0}(x)\right| \\
& \quad+C_{N}\left((R+M T)^{N} \underset{\substack{T x-x_{0} \mid<R+M T \\
T V}}{ }\left(v^{0}\right) T\|g-f-(g-f)(0)\|_{L^{\infty}}\right)^{1 / 2},
\end{aligned}
$$

with $M=\max (\operatorname{lip}(f), \operatorname{lip}(g))$.

The estimate (i) is known (see C.M. Dafermos [8], B.J. Lucier [14]), but the much stronger estimates (ii) and (iii) seem fairly new.

Proof of Theorem 3.1. For $k \in \mathbb{R}$ we have

$$
\begin{aligned}
& \partial_{t}|v-k|+\operatorname{div} \operatorname{sgn}(v-k)[f(v)-f(k)] \leq 0, \\
& \partial_{t}|u-k|+\operatorname{div} \operatorname{sgn}(u-k)[g(u)-g(k)] \leq 0 .
\end{aligned}
$$

Therefore, we can write

$$
\partial_{t}|u-k|+\operatorname{div} \operatorname{sgn}(u-k)[f(u)-f(k)] \leq \operatorname{div} \gamma_{k}(u),
$$

with

$$
\begin{aligned}
\gamma_{k}(\xi)= & \operatorname{sgn}(\xi-k)[(f-g)(\xi)-(f-g)(k)] \\
& -\operatorname{sgn}(-k)[(f-g)(0)-(f-g)(k)] \\
= & \operatorname{sgn}(\xi-k)[(f-g)(\xi)-(f-g)(0)] \\
& +[\operatorname{sgn}(\xi-k)-\operatorname{sgn}(-k)][(f-g)(0)-(f-g)(k)] .
\end{aligned}
$$

Notice that $\gamma_{k} \in \operatorname{lip}\left(\mathbb{R}, \mathbb{R}^{N}\right)$.

(i) Assume that $u^{0} \in B V\left(\mathbb{R}^{N}\right)$. Then

$$
\gamma_{k}(u) \in C\left(\left[0, \infty\left[, L_{l o c}^{1}\left(\mathbb{R}^{N}\right)\right) \cap \mathcal{B}\left(\left[0, \infty\left[, B V\left(\mathbb{R}^{N}\right)\right)\right.\right.\right.\right.
$$

(where $\mathcal{B}(X, Y)$ denotes the set of bounded functions $X \rightarrow Y$ ), and by Appendix A2

$$
\left|\frac{\partial}{\partial x_{i}} \gamma_{k}^{(j)}(u)\right| \leq \operatorname{lip}\left(\gamma_{k}^{(j)}\right)\left|\frac{\partial u}{\partial x_{i}}\right| \leq \operatorname{lip}(g-f)\left|\frac{\partial u}{\partial x_{i}}\right| .
$$


Therefore, $K_{k} \equiv \operatorname{div} \gamma_{k}(u) \in L^{\infty}(] 0, \infty\left[, \mathcal{M}\left(\mathbb{R}^{N}\right)\right)$ with

$$
\left|K_{k}\right| \leq \operatorname{lip}(g-f) \sum_{i=1}^{N}\left|\frac{\partial u}{\partial x_{i}}\right| \equiv \alpha_{K}
$$

We estimate $\alpha_{K}$ by

$$
\int_{\mathbb{R}^{N}} \alpha_{K}(t, d x)=\operatorname{lip}(g-f) T V(u(t, .)) \leq \operatorname{lip}(g-f) T V\left(u^{0}\right) .
$$

Now, the inequalities (1.3)-(1.4) are satisfied with a single term $K_{k}$ of order zero. Hence, we can apply Theorem 2.1. Then, we just let $\delta \rightarrow 0$, then $\Delta \rightarrow 0$ and finally $R \rightarrow \infty$ in (2.4), and we obtain

$$
\int_{\mathbb{R}^{N}}|u(T, .)-v(T, .)| \leq \int_{\mathbb{R}^{N}}\left|u^{0}-v^{0}\right|+C T \operatorname{lip}(g-f) T V\left(u^{0}\right) .
$$

The estimate (i) follows by exchanging $u$ and $v$.

(ii) The right-hand side of (3.6) can also be viewed as div $H_{k}$, with

$$
H_{k}=\gamma_{k}(u)
$$

We have

$$
\begin{aligned}
\left|\gamma_{k}(\xi)\right| & \leq|(f-g)(\xi)-(f-g)(0)|+2 \mathbb{I}_{|k| \leq|\xi|}|(f-g)(0)-(f-g)(k)| \\
& \leq Q(f, g)|\xi|+2 \mathbb{I}_{|k| \leq|\xi|} Q(f, g)|k| \\
& \leq 3 Q(f, g)|\xi| .
\end{aligned}
$$

Therefore, we get $\left|H_{k}\right| \leq 3 Q(f, g)|u| \equiv \alpha_{H}$. Since $u^{0} \in L^{1}\left(\mathbb{R}^{N}\right)$, we find that

$$
\int_{\mathbb{R}^{N}} \alpha_{H}(t, x) d x=3 Q(f, g) \int_{\mathbb{R}^{N}}|u(t, x)| d x \leq 3 Q(f, g)\left\|u^{0}\right\|_{L^{1}} .
$$

Now we can apply Theorem 2.1 with a single divergence term in the right-hand side. Since $v^{0} \in B V\left(\mathbb{R}^{N}\right)$, we can use (2.9). We let $\delta \rightarrow 0$, and, for any $\Delta>0$, we get

$$
\int_{\left|x-x_{0}\right|<R}|u(T, .)-v(T, .)| \leq \int_{\mathbb{R}^{N}}\left|u^{0}-v^{0}\right|+C_{N}\left(T V\left(v^{0}\right) \Delta+\frac{1}{\Delta} Q(f, g)\left\|u^{0}\right\|_{L^{1}} T\right) .
$$

Finally, we let $R$ tend to $\infty$, we choose the optimal $\Delta$, and we obtain (ii).

(iii) This estimate is a localized version of (ii), but we have to be careful in order to get the right domain of dependence. Assume that $v^{0} \in B V_{l o c}\left(\mathbb{R}^{N}\right)$, and let us denote $\widehat{R}=R+M T$ and $\widehat{B}=B\left(x_{0}, \widehat{R}\right)$. There exists an extension $\widehat{v}^{0}$ of $v^{0} \mid \widehat{B}$ such that $\widehat{v}^{0} \in L^{1} \cap B V\left(\mathbb{R}^{N}\right)$, supp $\widehat{v}^{0} \subset B\left(x_{0}, 2 \widehat{R}\right)$, and

$$
\begin{gathered}
\left\|\widehat{v}^{0}\right\|_{L^{1}\left(\mathbb{R}^{N}\right)} \leq C_{N}\left\|v^{0}\right\|_{L^{1}(\widehat{B})}, \\
T V_{\mathbb{R}^{N}}\left(\widehat{v}^{0}\right) \leq C_{N}\left(T V_{\widehat{B}}\left(v^{0}\right)+\frac{1}{\widehat{R}}\left\|v^{0}\right\|_{L^{1}(\widehat{B})}\right) .
\end{gathered}
$$

Let us define

$$
\widehat{u}^{0}(x)=\left\{\begin{array}{lll}
u^{0}(x) & \text { if } & x \in \widehat{B} \\
\widehat{v}^{0}(x) & \text { if } & x \notin \widehat{B}
\end{array}\right.
$$


and consider the entropy solutions $\widehat{u}$ and $\widehat{v}$ associated with the flux functions $g$ and $f$, and with initial data $\widehat{u}^{0}$ and $\widehat{v}^{0}$. Since supp $\widehat{u}^{0} \subset B\left(x_{0}, 2 \widehat{R}\right)$, for any $t \geq 0$ we have

$$
\operatorname{supp} \widehat{u}(t, .) \subset \bar{B}\left(x_{0}, 2 \widehat{R}+t \operatorname{lip}(g)\right) .
$$

Therefore, for any $t \geq 0$

$$
\left|\gamma_{k}(\widehat{u}(t, x))\right| \leq 3\|g-f-(g-f)(0)\|_{L^{\infty}} \mathbb{I}_{\bar{B}\left(x_{0}, 2 \widehat{R}+t \operatorname{Lip}(g)\right)}(x) \equiv \alpha_{H}(t, x) .
$$

Then we apply Theorem 2.1 to $\widehat{u}$ and $\widehat{v}$ with $H_{k}=\gamma_{k}(\widehat{u})$, and by letting $\delta \rightarrow 0$ we see that for any $\Delta>0$

$$
\begin{aligned}
& \int_{\left|x-x_{0}\right|<R}|\widehat{u}(T, x)-\widehat{v}(T, x)| \leq \int_{\mathbb{R}^{N}}\left|\widehat{u}^{0}-\widehat{v}^{0}\right| \\
& \quad+C_{N}\left[T V\left(\widehat{v}^{0}\right) \Delta+\frac{T}{\Delta}\left|\bar{B}\left(x_{0}, 2 \widehat{R}+T \operatorname{lip}(g)\right)\right|\|g-f-(g-f)(0)\|_{L^{\infty}}\right] .
\end{aligned}
$$

Choosing the optimal $\Delta$ and taking into account the definition of $\widehat{u}^{0}$ and $\widehat{v}^{0}$ and the finite speed of propagation for $\widehat{u}$ and $\widehat{v}$, we get

$$
\begin{aligned}
& \int_{\left|x-x_{0}\right|<R}|u(T, x)-v(T, x)| \\
& \quad \leq \int_{\left|x-x_{0}\right|<\widehat{R}}\left|u^{0}-v^{0}\right|+C_{N}\left[\widehat{R}^{N} T V\left(\widehat{v}^{0}\right) T\|g-f-(g-f)(0)\|_{L^{\infty}}\right]^{1 / 2} .
\end{aligned}
$$

It remains to estimate $T V_{\mathbb{R}^{N}}\left(\widehat{v}^{0}\right)$. By the Poincaré-Wirtinger inequality we have

$$
\int_{\widehat{B}}\left|v^{0}-\frac{1}{|\widehat{B}|} \int_{\widehat{B}} v^{0}\right| \leq C_{N} \widehat{R} T V_{\widehat{B}}\left(v^{0}\right) .
$$

Therefore, by (3.11), the result holds as soon as $\int_{\widehat{B}} v^{0}=0$. If not, let $c=\int_{\widehat{B}} v^{0} /|\widehat{B}|$. The functions $u-c$ and $v-c$ are entropy solutions associated to the flux functions $g(\xi+c)-g(0)$ and $f(\xi+c)-f(0)$, and have initial data $u^{0}-c$ and $v^{0}-c$. Since $\int_{\widehat{B}}\left(v^{0}-c\right)=0$, the result holds for these functions, and so it holds for $u$ and $v$.

\section{The NONLINEAR DIFFUSION MODEL}

This section is devoted to the proof of Theorem 1.1.

Let us prove (i). Since $u \in \mathcal{B}\left(\left[0, \infty\left[, B V\left(\mathbb{R}^{N}\right)\right)(\mathcal{B}(X, Y)\right.\right.$ denotes the set of bounded functions $X \rightarrow Y)$, we have $g(u) \in \mathcal{B}\left(\left[0, \infty\left[, B V\left(\mathbb{R}^{N}\right)\right)\right.\right.$ for any Lipschitz continuous function $g$ by Appendix A2. Moreover, $\frac{\partial}{\partial x_{j}} g(u) \in L^{\infty}(] 0, \infty\left[, \mathcal{M}\left(\mathbb{R}^{N}\right)\right)$, and

$$
\left|\frac{\partial}{\partial x_{j}} g(u)\right| \leq \operatorname{lip}(g)\left|\frac{\partial u}{\partial x_{j}}\right|
$$

By choosing

$$
g_{k}(\xi)=|\phi(\xi)-\phi(k)|-|\phi(0)-\phi(k)|,
$$

we get

$$
\left|\frac{\partial}{\partial x_{j}} g_{k}(u)\right| \leq \operatorname{lip}(\phi)\left|\frac{\partial u}{\partial x_{j}}\right| \equiv \alpha_{H}^{(j)}
$$


Now the error term in (1.8) can be written as

$$
\Delta|\phi(u)-\phi(k)|=\operatorname{div} H_{k}, \quad H_{k}=\nabla\left[g_{k}(u)\right],
$$

and we have $\left|H_{k}^{(j)}\right| \leq \alpha_{H}^{(j)}$. Therefore we can apply Theorem 2.1 with this single error term. We choose $\nu=0$ and we let $\delta \rightarrow 0$, so that $E^{t} \rightarrow 0$. Then $E^{x}$ is estimated by (2.9), and

$$
E^{H} \leq \frac{T}{\Delta} V \operatorname{lip}(\phi)
$$

We hence obtain that for any $\Delta>0$

$$
\begin{aligned}
& \int_{\left|x-x_{0}\right|<R}|u(T, x)-v(T, x)| d x \\
& \quad \leq \int_{\left|x-x_{0}\right|<R+M T+\Delta}|u(0, x)-v(0, x)| d x+C\left(T V\left(v^{0}\right) \Delta+\frac{T}{\Delta} \operatorname{lip}(\phi) V\right) .
\end{aligned}
$$

We then let $R \rightarrow \infty$, and by choosing the optimal value of $\Delta$ we get (1.9).

Now let us prove (ii). We consider the error term in (1.8) as

$$
\Delta|\phi(u)-\phi(k)|=\sum_{1 \leq i, j \leq N} \frac{\partial^{2} L_{k}^{(i j)}}{\partial x_{i} \partial x_{j}}, \quad L_{k}^{(i j)}=\delta_{i j} g_{k}(u),
$$

with $g_{k}$ defined in (4.2). Since we have

$$
\left|L_{k}^{(i j)}\right| \leq \delta_{i j} Q|u| \equiv \alpha_{L}^{(i j)}
$$

we can apply Theorem 2.1. As above, we choose $\nu=0$ and let $\delta \rightarrow 0$, so that $E^{t} \rightarrow 0$. We have

$$
E^{L} \leq \frac{T}{\Delta^{2}} N Q U
$$

and $E^{x}$ is estimated by (2.9). Therefore we get that for any $\Delta>0$

$$
\begin{aligned}
& \int_{\left|x-x_{0}\right|<R}|u(T, x)-v(T, x)| d x \\
& \quad \leq \int_{\left|x-x_{0}\right|<R+M T+\Delta}|u(0, x)-v(0, x)| d x+C\left(T V\left(v^{0}\right) \Delta+\frac{T}{\Delta^{2}} N Q U\right) .
\end{aligned}
$$

We then let $R \rightarrow \infty$, and by choosing the optimal value of $\Delta$ we get (1.10).

In order to prove (iii) we need a preliminary result, which is another version of Theorem 2.1. It formalizes the method of B. Cockburn and P.-A. Gremaud [6] to replace the $B V$ regularity of $u$ by the $B V$ regularity of $v$.

Proposition 4.1. Under the same hypothesis as in Theorem 2.1, assume moreover that

$$
H_{k}=H(t, x, k), \quad L_{k}^{(i j)}=L^{(i j)}(t, x, k)
$$

are Borel functions satisfying

$$
\begin{gathered}
\left|H\left(t, x, k_{1}\right)-H\left(t, x, k_{2}\right)\right| \leq M_{H}\left|k_{1}-k_{2}\right|, \\
\left|L^{(i j)}\left(t, x, k_{1}\right)-L^{(i j)}\left(t, x, k_{2}\right)\right| \leq M_{L}^{(i j)}\left|k_{1}-k_{2}\right| .
\end{gathered}
$$

Assume also that $v \in \mathcal{B}\left(\left[0, \infty\left[{ }_{l o c}, B V_{l o c}\left(\mathbb{R}^{N}\right)\right)\right.\right.$. 
Then, we can replace $E^{H}$ and $E^{L}$ in (2.4) by $E_{*}^{H}$ and $E_{*}^{L}$, where

$$
E_{*}^{H}=\frac{1}{\Delta+\nu} \sum_{j=1}^{N} \iint_{\substack{x \in B_{t} \\ 0<t \leq T}} \alpha_{H}^{(j)}(t, x), \quad E_{*}^{L}=\frac{1}{\Delta(\Delta+\nu)} \sum_{1 \leq i, j \leq N} \iint_{\substack{x \in B_{t} \\ 0<t \leq T}} \alpha_{L}^{(i j)}(t, x),
$$

provided that we add the term $C\left(E^{\partial H}+E^{\partial L}\right)$, with

$$
E^{\partial H}=M_{H} \sum_{j=1}^{N} \iint_{\substack{x \in B_{0} \\ 0<t \leq T+\delta}}\left|\frac{\partial v}{\partial x_{j}}(t, x)\right|, \quad E^{\partial L}=\frac{1}{\Delta} \sum_{1 \leq i, j \leq N} M_{L}^{(i j)} \iint_{\substack{x \in B_{0} \\ 0<t \leq T+\delta}}\left|\frac{\partial v}{\partial x_{j}}(t, x)\right| .
$$

Moreover, if $a \leq v \leq b$, it is sufficient that (4.8) holds for $a \leq k_{1}, k_{2} \leq b$.

Notice that the only difference between $E^{H}$ and $E_{*}^{H}$ (respectively $E^{L}$ and $E_{*}^{L}$ ) is that we replace a factor $1 / \Delta$ by $1 /(\Delta+\nu)$, which is bounded when $\Delta \rightarrow 0$. Then for a local estimate we choose for $\nu$ a finite positive value, and for a global estimate we just let $\nu \rightarrow \infty$. The result also holds when considering error terms for $v$ as in Proposition 2.2.

Proof of Proposition 4.1. In the proof of Theorem 2.1, instead of using (1.4) in (2.11), we keep the terms $H(t, x, k)$ and $L^{(i j)}(t, x, k)$. Hence, the terms we have to estimate are

$$
\begin{gathered}
R^{H}=\iiint \int-H(t, x, v(s, y)) \cdot \nabla_{x} \varphi d s d t d x d y, \\
R^{L}=\iiint \int \sum_{i j} L^{(i j)}(t, x, v(s, y)) \frac{\partial^{2} \varphi}{\partial x_{i} \partial x_{j}} d s d t d x d y .
\end{gathered}
$$

They can be written $R^{H}=R^{\partial H}+R_{*}^{H}, \quad R^{L}=R^{\partial L}+R_{*}^{L}$, with

$$
\begin{gathered}
R^{\partial H}=\iiint \int H(t, x, v(s, y)) \cdot \nabla_{y} \varphi d s d t d x d y, \\
R^{\partial L}=\iiint \int \sum_{i j} L^{(i j)}(t, x, v(s, y)) \frac{\partial^{2} \varphi}{\partial y_{i} \partial y_{j}} d s d t d x d y,
\end{gathered}
$$

and $R_{*}^{H}, R_{*}^{L}$ take into account $\nabla_{x} \varphi+\nabla_{y} \varphi$ and $\frac{\partial^{2} \varphi}{\partial x_{i} \partial x_{j}}-\frac{\partial^{2} \varphi}{\partial y_{i} \partial y_{j}}$. Then we estimate $R_{*}^{H}$ and $R_{*}^{L}$ as in the proof of Theorem 2.1. The only difference is that we no longer have the terms corresponding to the maximal order of derivation for $\zeta$. Therefore, in (2.28) and (2.30) we retain only terms in $1 / \theta$ and $1 / \Delta \theta$ respectively, instead of $1 / \Delta$ and $1 / \Delta^{2}$ as before. We hence get

$$
\limsup _{\varepsilon \rightarrow 0}\left|R_{*}^{H}\right| \leq C E_{*}^{H}, \quad \limsup _{\varepsilon \rightarrow 0}\left|R_{*}^{L}\right| \leq C E_{*}^{L} .
$$

Now, in order to estimate $R^{\partial H}$ and $R^{\partial L}$, we notice that for fixed $s, t, x$, the function $v(s,$.$) belongs to B V_{l o c}$. By the Lipschitz conditions (4.8) and by Appendix A2, we obtain that $H(t, x, v(s,).) \in B V_{l o c}$ and $L^{(i j)}(t, x, v(s,).) \in B V_{l o c}$. Therefore, we 
integrate by parts to get

$$
\begin{gathered}
R^{\partial H}=\iiint \int-\varphi \operatorname{div}_{y}[H(t, x, v(s, y))], \\
R^{\partial L}=\iiint \int-\sum_{i j} \frac{\partial \varphi}{\partial y_{i}} \frac{\partial}{\partial y_{j}}\left[L^{(i j)}(t, x, v(s, y))\right] .
\end{gathered}
$$

Then, by Appendix A2 (i), or (ii) if $a \leq v \leq b$,

$$
\begin{gathered}
\left|R^{\partial H}\right| \leq \iiint \int \varphi \sum_{j=1}^{N} M_{H}\left|\frac{\partial v}{\partial x_{j}}(s, y)\right| \\
\left|R^{\partial L}\right| \leq \iiint \int \sum_{i j}\left|\frac{\partial \varphi}{\partial y_{i}}\right| M_{L}^{(i j)}\left|\frac{\partial v}{\partial x_{j}}(s, y)\right| .
\end{gathered}
$$

These terms are finally estimated as in the proof of Proposition 2.2, and we obtain

$$
\limsup _{\varepsilon \rightarrow 0}\left|R^{\partial H}\right| \leq E^{\partial H}, \quad \limsup _{\varepsilon \rightarrow 0}\left|R^{\partial L}\right| \leq C E^{\partial L},
$$

which ends the proof of Proposition 4.1.

Proof of Theorem 1.1 (iii). We begin as in the proof of (ii). We notice that $L_{k}^{(i j)}=$ $\delta_{i j} g_{k}(u)$ can be written $L_{k}^{(i j)}=L^{(i j)}(t, x, k)$, with

$$
L^{(i j)}(t, x, k)=\delta_{i j}[|\phi(u(t, x))-\phi(k)|-|\phi(0)-\phi(k)|] .
$$

Therefore, the Lipschitz condition (4.8) is fulfilled with $M_{L}^{(i j)}=2 \delta_{i j} \operatorname{lip}(\phi)$, and we can apply Proposition 4.1. We obtain

$$
\begin{aligned}
& \int_{\left|x-x_{0}\right|<R}|u(T, x)-v(T, x)| \\
& \quad \leq \int_{\left|x-x_{0}\right|<R+M T+\Delta+\nu}\left|u^{0}(x)-v^{0}(x)\right|+C\left(E^{t}+E^{x}+E^{\partial L}+E_{*}^{L}\right) .
\end{aligned}
$$

We have

$$
E_{*}^{L} \leq \frac{N Q}{\Delta(\Delta+\nu)} \iint_{] 0, T\left[\times \mathbb{R}^{N}\right.}|u(t, x)| d t d x, \quad E^{\partial L} \leq \frac{2(T+\delta)}{\Delta} \operatorname{lip}(\phi) T V\left(v^{0}\right),
$$

and $E^{x}$ is estimated by (2.9). By letting $\delta \rightarrow 0$ and $\nu \rightarrow \infty$ (so that $E_{*}^{L} \rightarrow 0$ ) we get for any $\Delta>0$

$$
\int_{\left|x-x_{0}\right|<R}|u(T, x)-v(T, x)| \leq \int_{\mathbb{R}^{N}}\left|u^{0}(x)-v^{0}(x)\right|+C\left(T V\left(v^{0}\right) \Delta+\frac{T}{\Delta} \operatorname{lip}(\phi) T V\left(v^{0}\right)\right) .
$$

By choosing the optimal value of $\Delta$ and letting $R \rightarrow \infty$ we obtain (iii). 


\section{A RELAXATION MODEL FOR Finite VOLUME METHODS}

In this section we use Theorem 2.1 to deduce an estimate in $h^{1 / 4}$ for the finite volume type model presented in the introduction. We first state an existence result.

Proposition 5.1. With the notations of the introduction, for any $\varepsilon>0$ and any $u^{0} \in L^{1}\left(\mathbb{R}^{N}\right)$ there exists a unique solution $u \in C\left(\left[0, \infty\left[, L^{1}\left(\mathbb{R}^{N}\right)\right)\right.\right.$ of $(1.15)$ satisfying the entropy inequalities (1.16) for all convex, Lipschitz and $C^{1}$ functions $S$, or equivalently for all Kružkov entropies.

Since this result uses very standard techniques, we only give a short sketch of the proof. First let us notice that for an arbitrary convex and Lipschitz $S, S^{\prime}(u)$ is not well defined because of possible jumps of $S^{\prime}$. That is why we require $S$ to be $C^{1}$. Then, if (1.16) holds for these $C^{1}$ test functions, it is easy to see that it also holds for the entropies $S_{k}$, with the convention that $S_{k}^{\prime}(\xi)=\operatorname{sgn}(\xi-k)$ and $\operatorname{sgn}(0)=0$.

The uniqueness is obtained by Kružkov's method. We actually get that for two solutions $u, v$ of (1.16),

$$
\begin{aligned}
\partial_{t}|u-v|+\operatorname{div} \operatorname{sgn}(u-v)[f(u)-f(v)] & \\
\leq & \left.\frac{\left|P^{0}(u-v)\right|-|u-v|}{\varepsilon} \text { in }\right] 0, \infty\left[\times \mathbb{R}^{N},\right.
\end{aligned}
$$

which yields the contraction property

$$
\|u(t, .)-v(t, .)\|_{L^{1}} \leq\left\|u^{0}-v^{0}\right\|_{L^{1}} .
$$

For existence, we use the small diffusion approximation method. Here the $L^{1}-$ $B V$ hypothesis (1.13) on the grid is involved. It is actually equivalent to asserting that $P^{0}$ maps $L^{1}\left(\mathbb{R}^{N}\right)$ into $B V\left(\mathbb{R}^{N}\right)$ with

$$
T V\left(P^{0} w\right) \leq K_{h}\|w\|_{L^{1}}, \quad w \in L^{1}\left(\mathbb{R}^{N}\right) .
$$

This yields the following a priori estimate for a solution $u$ of (1.15)-(1.16) of initial data $u^{0} \in L^{1} \cap B V\left(\mathbb{R}^{N}\right)$ :

$$
\left\|\frac{\partial u}{\partial x_{j}}(t, .)\right\|_{\mathcal{M}} \leq e^{-t / \varepsilon}\left\|\frac{\partial u^{0}}{\partial x_{j}}\right\|_{\mathcal{M}}+\left(1-e^{-t / \varepsilon}\right) K_{h}\left\|u^{0}\right\|_{L^{1}} .
$$

This allows us to prove existence for $u^{0} \in L^{1} \cap B V$. Then by the contraction property (5.2) we obtain the existence of a solution for any $u^{0} \in L^{1}\left(\mathbb{R}^{N}\right)$.

Now we can prove the convergence rate to the continuous solution.

Proof of Theorem 1.2. If $u^{0} \in L^{1} \cap L^{p}\left(\mathbb{R}^{N}\right)$ for some $p, 1 \leq p<\infty$, we can actually choose $S(\xi)=|\xi|^{p}$ in (1.16). Integrating this inequality with respect to $x$, we get

$$
\left.\frac{d}{d t} \int_{\mathbb{R}^{N}}|u|^{p} d x \leq \int_{\mathbb{R}^{N}} S^{\prime}(u) \frac{P^{0} u-u}{\varepsilon} d x \quad \text { in } \quad\right] 0, \infty[.
$$

Then, since $S^{\prime}\left(P^{0} u\right)$ is constant in each cell, and since the integral of $P^{0} u-u$ on each cell vanishes, we have

$$
\int_{\mathbb{R}^{N}} S^{\prime}(u) \frac{P^{0} u-u}{\varepsilon}=\int_{\mathbb{R}^{N}}\left[S^{\prime}(u)-S^{\prime}\left(P^{0} u\right)\right] \frac{P^{0} u-u}{\varepsilon} \leq 0 .
$$

Therefore, we obtain

$$
\left.\frac{d}{d t} \int_{\mathbb{R}^{N}}|u|^{p}+\int_{\mathbb{R}^{N}}\left[S^{\prime}\left(P^{0} u\right)-S^{\prime}(u)\right] \frac{P^{0} u-u}{\varepsilon} \leq 0 \quad \text { in } \quad\right] 0, \infty[
$$


and for any $T \geq 0$

$$
\int_{\mathbb{R}^{N}}|u(T, .)|^{p}+\iint_{] 0, T\left[\times \mathbb{R}^{N}\right.}\left[S^{\prime}\left(P^{0} u\right)-S^{\prime}(u)\right] \frac{P^{0} u-u}{\varepsilon} \leq \int_{\mathbb{R}^{N}}\left|u^{0}\right|^{p} .
$$

In the case where $u^{0} \in L^{1} \cap L^{2}\left(\mathbb{R}^{N}\right)(p=2)$, we find that

$$
\iint_{] 0, \infty\left[\times \mathbb{R}^{N}\right.} \frac{\left(P^{0} u-u\right)^{2}}{\varepsilon} \leq \int_{\mathbb{R}^{N}} \frac{\left|u^{0}\right|^{2}}{2} .
$$

The announced inequality (1.17) easily follows by Hölder's inequality. Now we write the entropy inequalities, for $k \in \mathbb{R}$

$$
\begin{aligned}
\partial_{t}|u-k| & +\operatorname{div} \operatorname{sgn}(u-k)[f(u)-f(k)] \\
& \leq \operatorname{sgn}(u-k) \frac{P^{0} u-u}{\varepsilon} \\
& =\left[\operatorname{sgn}(u-k)-\operatorname{sgn}\left(P^{0} u-k\right)\right] \frac{P^{0} u-u}{\varepsilon}+\operatorname{sgn}\left(P^{0} u-k\right) \frac{P^{0} u-u}{\varepsilon} \\
& \leq \operatorname{sgn}\left(P^{0} u-k\right) \frac{P^{0} u-u}{\varepsilon} .
\end{aligned}
$$

Since the integral in each cell of the right-hand side vanishes (for a fixed $t$ ), we can invert the "div" operator as in Appendix A1, and therefore

$$
\operatorname{sgn}\left(P^{0} u-k\right) \frac{P^{0} u-u}{\varepsilon}=\operatorname{div} H_{k}
$$

for some $H_{k} \in L^{\infty}(] 0, \infty\left[, L^{1}\left(\mathbb{R}^{N}\right)\right)$, which can be bounded above by (A.7) and (1.17):

$$
\iint_{0, T\left[\times B\left(x_{0}, R\right)\right.}\left|H_{k}(t, x)\right| d t d x \leq h \sqrt{\frac{T\left|B\left(x_{0}, R+2 h\right)\right|}{2 \varepsilon}}\left\|u^{0}\right\|_{L^{2}} .
$$

By examining the proof in Appendix A1, it is easy to see that since the sign in (5.9) is bounded by 1 , we have indeed

$$
\left|H_{k}(t, x)\right| \leq \alpha_{H}(t, x)
$$

for some $\alpha_{H}$ independent of $k$, and which also satisfies

$$
\iint_{0, T\left[\times\left(x_{0}, R\right)\right.} \alpha_{H}(t, x) d t d x \leq h \sqrt{\frac{T\left|B\left(x_{0}, R+2 h\right)\right|}{2 \varepsilon}}\left\|u^{0}\right\|_{L^{2}} .
$$

Therefore, combining (5.8) and (5.9), we may apply Theorem 2.1 with the sole error term (5.9). By choosing $\nu=0$ and letting $\delta \rightarrow 0$ (so that $E^{t} \rightarrow 0$ ) we obtain, using (2.9) for $E^{x}$ and (5.11) for $E^{H}$, that for any $\Delta>0$

$$
\begin{aligned}
\int_{\left|x-x_{0}\right|<R}|u(T, x)-v(T, x)| d x \leq & \int_{\left|x-x_{0}\right|<R+M T+\Delta}\left|u^{0}(x)-v^{0}(x)\right| d x \\
& +C_{N}\left(T V\left(v^{0}\right) \Delta+\frac{h}{\Delta} \sqrt{\frac{T(R+M T+\Delta+h)^{N}}{\varepsilon}}\left\|u^{0}\right\|_{L^{2}}\right) .
\end{aligned}
$$


Finally, we choose

$$
\Delta=\sqrt{\widehat{R} \ell}
$$

and we get the inequality (1.18).

Remark. It is an open problem to obtain an estimate in $h^{1 / 2}$ (except in one dimension see R. Sanders [17]). Notice that Proposition 4.1 cannot be applied, since the term $H_{k}$ is not Lipschitz continuous in $k$. When all the cells are star-shaped, denoting by $H(t, x)$ the function obtained Appendix A1 such that

$$
\operatorname{div} H=\frac{P^{0} u-u}{\varepsilon},
$$

we actually have, by the support property (A.4),

$$
H_{k}=\operatorname{sgn}\left(P^{0} u-k\right) H .
$$

So in this case the bound (5.11) is obvious.

$$
\begin{aligned}
& \text { APPENDIX. INVERSION OF THE "DIV" OPERATOR; } \\
& \text { COMPOSITION OF LIPSCHITZ AND BV FUNCTIONS }
\end{aligned}
$$

A1. Inversion of the "div" operator. We consider a question arising from the finite volume type of approximations in $\S 5$. In this context, we need the result below in order to use the error estimates stated in $\S 2$.

Lemma A1.1. Let $\left(C_{i}\right)_{i \in I}$ be a general grid of $\mathbb{R}^{N}$ as described in the introduction. Assume that $m \in L^{1}\left(\mathbb{R}^{N}\right)$ satisfies

$$
\int_{C_{i}} m(x) d x=0, \quad i \in I .
$$

Then there exists a vector field $H(x)$ such that

$$
\begin{gathered}
\operatorname{div} H=m \quad \text { in } \quad \mathbb{R}^{N}, \\
\|H\|_{L^{1}\left(\mathbb{R}^{N}\right)} \leq h\|m\|_{L^{1}\left(\mathbb{R}^{N}\right)} .
\end{gathered}
$$

Remark A1.2. (1) If all the cells $C_{i}$ are star-shaped (there is a point $x_{i} \in C_{i}$ such that for any $x \in C_{i}$ the segment $\left[x_{i}, x\right]$ is included in $C_{i}$ ), then the construction of $H$ is purely local. More precisely,

$$
H=\sum_{i \in I} H_{i}, \quad H_{i}=0 \quad \text { in } \quad \mathbb{R}^{N} \backslash C_{i},
$$

with

$$
\begin{gathered}
\operatorname{div} H_{i}=m \mathbb{I}_{C_{i}}, \\
\left\|H_{i}\right\|_{L^{1}\left(\mathbb{R}^{N}\right)} \leq h\left\|m \mathbb{I}_{C_{i}}\right\|_{L^{1}\left(\mathbb{R}^{N}\right)} .
\end{gathered}
$$

(2) The estimate (A.3) can be localized: for any bounded Borel set $\omega \subset \mathbb{R}^{N}$, we have

$$
\begin{gathered}
\|H\|_{L^{1}(\omega)} \leq h\|m\|_{L^{1}\left(\omega^{h}\right)}, \\
\omega^{h}=\bigcup_{\operatorname{dist}\left(C_{i}, \omega\right) \leq h} C_{i} .
\end{gathered}
$$


Actually, $H_{i}=0$ in $\omega$ as soon as $\operatorname{dist}\left(C_{i}, \omega\right)>h$, with $H_{i}$ defined in the proof below.

Proof of Lemma A1.1. To solve (A.2) in the distributional sense means that we look for a function $H$ such that, for all test functions $\varphi \in C_{c}^{\infty}\left(\mathbb{R}^{N}\right)$,

$$
\begin{aligned}
& -\int_{\mathbb{R}^{N}} H(x) \cdot \nabla \varphi(x) d x=\int_{\mathbb{R}^{N}} m(x) \varphi(x) d x \\
& \quad=\sum_{i \in I} \int_{C_{i}} m(x)\left(\varphi(x)-\varphi\left(x_{i}\right)\right) d x \\
& \quad=\sum_{i \in I} \int_{C_{i}} m(x) \int_{0}^{1} \nabla \varphi\left(x_{i}+\theta\left(x-x_{i}\right)\right) \cdot\left(x-x_{i}\right) d \theta d x \\
& =\sum_{i \in I} \int_{\mathbb{R}^{N}} \int_{0}^{1}\left(m \mathbb{I}_{C_{i}}\right)\left(x_{i}+\frac{y-x_{i}}{\theta}\right) \nabla \varphi(y) \cdot \frac{y-x_{i}}{\theta} d \theta \frac{d y}{\theta^{N}} .
\end{aligned}
$$

Here $x_{i}$ denotes an arbitrary point of $C_{i}$, but in the star-shaped case it should possess the property in Remark A1.2 (1). Therefore we choose

$$
\begin{gathered}
H=\sum_{i \in I} H_{i}, \\
H_{i}(y)=-\int_{0}^{1}\left(m \mathbb{I}_{C_{i}}\right)\left(x_{i}+\frac{y-x_{i}}{\theta}\right) \frac{y-x_{i}}{\theta^{N+1}} d \theta .
\end{gathered}
$$

We may majorize the $L^{1}$ norm of $H_{i}$,

$$
\begin{aligned}
\int_{\mathbb{R}^{N}}\left|H_{i}(y)\right| d y & \leq \int_{\mathbb{R}^{N}} \int_{0}^{1}\left(|m| \mathbb{I}_{C_{i}}\right)\left(x_{i}+\frac{y-x_{i}}{\theta}\right) \frac{\left|y-x_{i}\right|}{\theta^{N+1}} d \theta d y \\
& =\int_{0}^{1} \int_{\mathbb{R}^{N}}\left(|m| \mathbb{I}_{C_{i}}\right)(x)\left|x-x_{i}\right| d x d \theta \\
& \leq \operatorname{diam}\left(C_{i}\right) \int_{C_{i}}|m(x)| d x
\end{aligned}
$$

Adding these inequalities, we obtain the statement of Lemma A1.1. In the starshaped case, we notice from formula (A.11) that if $H_{i}(y) \neq 0$, then $z_{\theta}=x_{i}+\frac{y-x_{i}}{\theta} \in$ $C_{i}$ for some $\left.\theta \in\right] 0,1\left[\right.$. This means that $y=x_{i}+\theta\left(z_{\theta}-x_{i}\right)$ also belongs to $C_{i}$. This proves (A.4). The properties (A.5) and (A.6) are contained in the above proof.

A2. Composition of Lipschitz and $B V$ functions. Let $\Omega$ be an open subset of $\mathbb{R}^{N}$ and consider a function $u \in B V_{\text {loc }}(\Omega)$, and $g: \mathbb{R} \rightarrow \mathbb{R}$ Lipschitz continuous. The well-known theory of A.I. Vol'pert [20] allows us to compute the gradient of $g(u)$ provided that $g \in C^{1}$. However, for a general Lipschitz function $g$ we have the following simple result.

Lemma A2.1. With the above notations, $g(u)$ belongs to $B V_{l o c}(\Omega)$ and:

(i) in the sense of measures,

$$
\left|\frac{\partial}{\partial x_{j}}[g(u)]\right| \leq \operatorname{lip}(g)\left|\frac{\partial u}{\partial x_{j}}\right| .
$$

(ii) if $a \leq u \leq b$, then

$$
\left|\frac{\partial}{\partial x_{j}}[g(u)]\right| \leq \operatorname{lip}_{[a, b]}(g)\left|\frac{\partial u}{\partial x_{j}}\right| .
$$


Proof. Let us define $g_{n}=\rho_{n} * g, u_{n}=\rho_{n} * u$, where $\rho_{n} \geq 0$ is a standard smoothing sequence (in 1 or $N$ dimensions). Since these functions are smooth, we have

$$
\left|\frac{\partial}{\partial x_{j}} g_{n}\left(u_{n}\right)\right|=\left|g_{n}^{\prime}\left(u_{n}\right) \frac{\partial u_{n}}{\partial x_{j}}\right| \leq\left\|g_{n}^{\prime}\right\|_{L^{\infty}}\left|\frac{\partial u_{n}}{\partial x_{j}}\right| \leq \operatorname{lip}(g)\left|\frac{\partial u_{n}}{\partial x_{j}}\right| .
$$

From the formula $\frac{\partial u_{n}}{\partial x_{j}}=\rho_{n} * \frac{\partial u}{\partial x_{j}}$ we get $\left|\frac{\partial u_{n}}{\partial x_{j}}\right| \leq \rho_{n} *\left|\frac{\partial u}{\partial x_{j}}\right|$, and hence

$$
\left|\frac{\partial}{\partial x_{j}} g_{n}\left(u_{n}\right)\right| \leq \operatorname{lip}(g) \rho_{n} *\left|\frac{\partial u}{\partial x_{j}}\right| \text {. }
$$

Therefore, for any test function $\varphi \in C_{c}^{\infty}(\Omega)$ we have

$$
\left|\left\langle\frac{\partial}{\partial x_{j}}\left[g_{n}\left(u_{n}\right)\right], \varphi\right\rangle\right| \leq \operatorname{lip}(g)\left\langle\rho_{n} *\left|\frac{\partial u}{\partial x_{j}}\right|,|\varphi|\right\rangle,
$$

and by letting $n \rightarrow \infty$

$$
\left|\left\langle\frac{\partial}{\partial x_{j}}[g(u)], \varphi\right\rangle\right| \leq \operatorname{lip}(g)\left\langle\left|\frac{\partial u}{\partial x_{j}}\right|,|\varphi|\right\rangle .
$$

Since the right-hand side is bounded by $C\|\varphi\|_{L^{\infty}}$, we get that $\frac{\partial}{\partial x_{j}}[g(u)]$ is locally a measure, and hence $g(u) \in B V_{l o c}(\Omega)$. Then by density (A.16) still holds for any $\varphi \in C_{c}(\Omega)$. By approximation in $L^{1}\left(\left|\partial_{j}[g(u)]\right|+\left|\partial_{j} u\right|\right)$, (A.16) is also true for any $\varphi$ measurable and bounded with compact support in $\Omega$. Therefore,

$$
\left|\int_{E} \frac{\partial}{\partial x_{j}}[g(u)]\right| \leq \operatorname{lip}(g) \int_{E}\left|\frac{\partial u}{\partial x_{j}}\right|
$$

for any Borel set $E$ with compact closure in $\Omega$, and (A.13) follows from the definition of the absolute value of a measure.

In order to prove (ii) we define

$$
\widetilde{g}(\xi)=\left\{\begin{array}{lll}
g(\xi) & \text { if } \quad a \leq \xi \leq b, \\
g(a) & \text { if } \quad \xi \leq a \\
g(b) & \text { if } \quad \xi \geq b
\end{array}\right.
$$

Then $\operatorname{lip}(\widetilde{g})=\operatorname{lip}_{[a, b]}(g)$, and $\widetilde{g}(u)=g(u)$. We get the result by applying (i) to $\widetilde{g}$ and $u$.

\section{REFERENCES}

1. Ph. Benilan, R. Gariepy, Strong solutions in $L^{1}$ of degenerate parabolic equations, J. Diff. Eq. 119 (1995), 473-502. MR 96c:35078

2. F. Bouchut, Ch. Bourdarias, B. Perthame, A MUSCL method satisfying all the numerical entropy inequalities, Math. Comp. 65 (1996), 1439-1461. MR 97a:65080

3. S. Champier, T. Gallouët, R. Herbin, Convergence of an upstream finite volume scheme for a nonlinear hyperbolic equation on a triangular mesh, Numer. Math. 66 (1993), 139-157. MR 95b:65117

4. B. Cockburn, F. Coquel, P. Le Floch, An error estimate for finite volume methods for multidimensional conservation laws, Math. Comp. 63 (1994), 77-103. MR 95d:65078

5. B. Cockburn, F. Coquel, P. Le Floch, Convergence of the finite volume method for multidimensional conservation laws, SIAM J. Numer. Anal. 32 (1995), 687-705. MR 97f:65051

6. B. Cockburn, P.-A. Gremaud, A priori error estimates for numerical methods for scalar conservation laws. Part I: The general approach, Math. Comp. 65 (1996), 533-573. MR 96g:65089

7. B. Cockburn, P.-A. Gremaud, Error estimates for finite element methods for scalar conservation laws, SIAM J. Numer. Anal. 33 (1996), 522-554. MR 97e:65096 
8. C.M. Dafermos, Polygonal approximations of solutions of the initial value problem for a conservation law, J. Math. Anal. Appl. 38 (1972), 33-41. MR 46:2210

9. R. Eymard, T. Gallouët, R. Herbin, The finite volume method, to appear in Handbook of Numerical Analysis, Ph. Ciarlet and J.L. Lions, eds.

10. S.N. Kružkov, First order quasilinear equations in several independent variables, Math. USSR Sb. 10 (1970), 217-243. MR 42:2159

11. N.N. Kuznetsov, Accuracy of some approximate methods for computing the weak solutions of a first-order quasi-linear equation, USSR Comp. Math. and Math. Phys. 16 (1976), no. 6, 105-119. MR 58:3510

12. P.D. Lax, Hyperbolic systems of conservation laws II, Comm. Pure Appl. Math. 10 (1957), 537-566. MR 20:1761

13. T.-P. Liu, M. Pierre, Source-solutions and asymptotic behavior in conservation laws, J. Diff. Eq. 51 (1984), 419-441. MR 85i:35094

14. B.J. Lucier, A moving mesh numerical method for hyperbolic conservation laws, Math. Comp. 46 (1986), 59-69. MR 87m:65141

15. O.A. Oleinik, On the uniqueness and stability of the generalized solution of the Cauchy problem for a quasilinear equation, A.M.S. Transl. (2) 33 (1963), 285-290. MR 22:8187

16. B. Perthame, Convergence of $N$-schemes for linear advection equations, Trends in Applications of Mathematics to Mechanics, Pitman Mono. Ser. in Pure and Applied Math. 77, New-York (1995).

17. R. Sanders, On convergence of monotone finite difference schemes with variable spatial differencing, Math. Comp. 40 (1983), 91-106. MR 84a:65075

18. A. Szepessy, Convergence of a shock-capturing streamline diffusion finite element method for scalar conservation laws in two space dimensions, Math. Comp. 53 (1989), 527-545. MR 90h: 65156

19. J.-P. Vila, Convergence and error estimates in finite volume schemes for general multidimensional scalar conservation laws I. Explicit monotone schemes, RAIRO Model. Math. Anal. Numér. 28 (1994), 267-295. MR 96d:65150

20. A.I. Vol'pert, The spaces $B V$ and quasilinear equations, Math. USSR Sbornik 2 (1967), 225267. MR 35:7172

Université D'Orléans et CNRS, UMR 6628, Dépt. De Mathématiques, BP 6759, 45067 ORLÉANS CEDEX 2, France

E-mail address: fbouchut@labomath.univ-orleans.fr

Université Pierre et Marie Curie, Laboratoire D'Analyse Numérique et URA 189, BC 187, 4 Place Jussieu, 75252 Paris Cedex 05, France

E-mail address: perthame@ann.jussieu.fr 